\title{
Análise das Quantidades de Óleo e Sal de Adição em Refeições Escolares
}

\author{
Roseane Moreira Sampaio Barbosa ${ }^{1}$, Nathalia Cardoso Oliveira ${ }^{2}$, \\ Daniele Mendonça Ferreira ${ }^{3}$, Patrícia Camacho Dias ${ }^{4}$, Silvia Pereira ${ }^{5}$, \\ Daniele da Silva Bastos Soares ${ }^{6}$, Patrícia Henriques ${ }^{7}$
}

\begin{abstract}
RESUMO
O presente estudo teve o objetivo de verificar as quantidades de sal e óleo de adição nas refeições "almoço" ofertadas em uma escola municipal do Estado do Rio de Janeiro e comparar com a recomendação do Programa Nacional de Alimentação Escolar - PNAE. Inicialmente foram determinadas as quantidades utilizadas de sal e óleo às preparações alimentares das refeições, a partir da diferença entre o peso das quantidades de sal e óleo inicial e final diário disponível para uso na Unidade de Alimentação e Nutrição Escolar (Uane). Após o preparo, pesou-se o rendimento total das preparacõos alimentares e foi estabelecido o percentual de sal/óleo utilizados no total de preparações presentes nas refeições produzidas naquele dia. Em seguida, foi determinado o peso da porção da refeição ofertada aos escolares e, então, aplicado o percentual determinado anteriormente e calculada a quantidade de sal/óleo adicionados nas refeições oferecidas aos escolares. Foram encontrados como média 3,05g de sal por refeição, estando acima do que é preconizado pelo PNAE, e em relação às gorduras totais a porção apresentou-se acima da recomendação por refeição, $7,5 \mathrm{~g}$, com o valor médio de $12,54 \mathrm{~g} /$ refeição. Concluiu-se que existe a necessidade de redução das quantidades de sódio e óleo de adição no preparo das refeições que são distribuídas aos escolares, e que estas devem ser padronizadas para não superar o limite de $1 \mathrm{~g}$ de sal/refeição/aluno e manter o limite recomendável de lipídios.
\end{abstract}

Palavras-chave: Alimentação escolar. Sódio. Gorduras na dieta. Almoço.

\section{ANALYSE OF THE AMOUNT OF OIL AND SALT ADDED IN SCHOOL MEALS}

\section{ABSTRACT}

The present study had the objective of verifying the amounts of salt and oil added in the "lunch" meals offered in a municipal school of the state of Rio de Janeiro and compare with the recommendation of the PNAE (Brazilian School Nutrition Program). The amounts of salt and oil used in the meal preparations were initially determined from the difference between the weight of the initial and final daily salt and oil amounts available for use in Food and Nutrition Unit schools. After preparation, the total yield of the food preparations was weighed and the percentage of salt/oil used in all preparations present in the meals produced that day was established. Then, the weight of the portion of the meal offered to the schoolchildren was determined and then the percentage determined previously was applied and the amount of salt / oil added in the meals offered to the schoolchildren was calculated. An average of $3,05 \mathrm{~g}$ of salt per meal was found, higher than what is recommended by PNAE, and in relation to total fat, the portion was above the recommendation per meal, $7,5 \mathrm{~g}$, with a mean value of $12,54 \mathrm{~g} /$ meal. It was concluded that is needed to reduce the amounts of sodium and oil in the preparation of meals that are distributed to schoolchildren and that these should be standardized to not exceed the limit of $1 \mathrm{~g}$ of salt/meal/schoolchildren and maintain the recommended lipid limit.

Keywords: School feeding. Sodium. Diet fats. Lunch.

RECEBIDO EM: 1이/2019

MODIFICAÇÕES SOLICITADAS EM: 28/1/2020

ACEITO EM: 5/4/2020

\footnotetext{
Doutorado em Nutrição/UFRJ. Grupo de Ensino, Pesquisa e Extensão em Alimentação e Saúde do Escolar (http://www.nutricao.uff.br/content/gepase) - Departamento de Nutrição Social/Faculdade de Nutrição Emília de Jesus Ferreiro/Universidade Federal Fluminense. http://lattes.cnpq.br/4211691428243654. https://orcid.org/0000-0002-0850-7143. roseanesampaio@id.uff.br

Graduação em Nutrição/UFF. Grupo de Ensino, Pesquisa e Extensão em Alimentação e Saúde do Escolar (http://www.nutricao.uff.br/content/gepase) Faculdade de Nutrição Emília de Jesus Ferreiro/Universidade Federal Fluminense. http://lattes.cnpq.br/5095838107857448. https://orcid.org/0000-00016638-4818. seapereira@icloud.com

Doutorado em Ciências dos Alimentos/UFRJ. Grupo de Ensino, Pesquisa e Extensão em Alimentação e Saúde do Escolar (http://www.nutricao.uff.br/ content/gepase) - Departamento de Nutrição Social/Faculdade de Nutrição Emília de Jesus Ferreiro/Universidade Federal Fluminense. http://lattes.cnpq. br/1163585343443325. https://orcid.org/0000-0001-5196-9055. daniele_ferreira@id.uff.br

Doutorado em Política Social/UFF. Grupo de Ensino, Pesquisa e Extensão em Alimentação e Saúde do Escolar (http://www.nutricao.uff.br/content/ gepase) - Departamento de Nutrição Social/Faculdade de Nutrição Emília de Jesus Ferreiro/Universidade Federal Fluminense. http://lattes.cnpq. br/2032672242741923. https://orcid.org/0000-0002-0674-8832. diaspec2@gmail.com

Doutorado em Ciências dos Alimentos/UFRJ. Grupo de Ensino, Pesquisa e Extensão em Alimentação e Saúde do Escolar (http://www.nutricao.uff.br/ content/gepase) - Departamento de Nutrição Social/Faculdade de Nutrição Emília de Jesus Ferreiro/Universidade Federal Fluminense. http://lattes.cnpq. br/9062811018583569. https://orcid.org/0000-0003-1538-4097.seapereira@gmail.com

Doutorado em Ciências dos Alimentos/UFRJ. Grupo de Ensino, Pesquisa e Extensão em Alimentação e Saúde do Escolar (http://www.nutricao.uff.br/ content/gepase) - Departamento de Nutrição Social/Faculdade de Nutrição Emília de Jesus Ferreiro/Universidade Federal Fluminense. http://lattes.cnpq. br/1163585343443325. https://orcid.org/0000-0001-5196-9055.danielebastos@id.uff.br

Doutorado em Saúde Pública ENSP/Fiocruz. Grupo de Ensino, Pesquisa e Extensão em Alimentação e Saúde do Escolar (http://www.nutricao.uff.br/ content/gepase) - Departamento de Nutrição Social/Faculdade de Nutrição Emília de Jesus Ferreiro/Universidade Federal Fluminense. http://lattes.cnpq. br/6889568909022390.https://orcid.org/0000-001-8154-0962. patihenriques@gmail.com
} 


\section{INTRODUÇÃO}

Atualmente, constata-se o consumo de alimentos ultraprocessados, ricos em açúcares, gorduras e sal e redução do consumo de legumes, vegetais e frutas, contribuindo para o aumento da tendência de sobrepeso e obesidade em crianças e adolescentes (SOUZA et al., 2016). A Organização Mundial da Saúde (OMS) recomenda para crianças o consumo diário de menos de $2 \mathrm{~g}$ de sódio, incluindo a ingestão do sódio intrínseco nos alimentos (WHO, 2015). No Brasil, a Pesquisa Nacional de Orçamentos Familiares 2008-2009 (POF) mostrou que a quantidade média de sódio consumido nos domicílios é de 4,7g por pessoa, excedendo, assim, em mais de duas vezes o limite recomendado (IBGE, 2011; MILL et al., 2019).

A preferência por sal aparece já na infância, a partir dos 4 meses de idade, e pode estar relacionada à sua exposição alimentar (JACKIX et al., 2014). A presença de açúcares, sal e gorduras nos alimentos, de modo geral, está associada à melhor aceitação da dieta e, por isso, estes alimentos acabam sendo os favoritos entre o público infantil (BOUHLAL; ISSANCHOU; NICKLAUS, 2011; LOUZADA et al., 2015). Considerando a maior aceitação das preparações quando adicionados estes ingredientes, muitas vezes os responsáveis pelo preparo das refeições podem ser induzidos a acrescentar elevadas quantidades destes às preparações alimentares realizadas nas escolas (JACKIX et al., 2014; SILVA; COSTA; GIUGLIANI, 2016).

Nas Unidades de Alimentação e Nutrição Escolar (Uane) públicas, as merendeiras são as responsáveis pelo preparo das refeições de acordo com os cardápios planejados pelo Nutricionista. A ausência do profissional diariamente na Uane e de Fichas Técnicas de Preparo (FTP), entretanto, permite que elas decidam, muitas vezes, pela eliminação e/ou substituição de um ingrediente e/ou preparação alimentar, além da alteração em sua forma de preparo (SANTOS; SREBERMICH, 2015; MENEZES; SANTANA; NASCIMENTO, 2018). Sendo assim, podem ocorrer adaptações no cardápio que resultem na adição excessiva de óleo e sal às preparações alimentares, ultrapassando os limites estabelecidos nas recomendações do Programa Nacional de Alimentação Escolar (PNAE) (BRASIL, 2013).

O PNAE tem como objetivo contribuir para o crescimento, desenvolvimento, aprendizagem, rendimento escolar e formação de hábitos alimentares saudáveis, por meio da oferta da alimentação escolar que atinja as necessidades nutricionais dos estudantes durante sua permanência na escola e de ações de educação alimentar e nutricional. Desta forma, as merendeiras devem ser orientadas quanto à correta adição de sal e óleo às preparações alimentares, pois, segundo o PNAE, a oferta da alimentação escolar deve conter de $15 \%$ a $30 \%$ de gorduras totais do valor energético total (VET) e um grama de sal quando ofertada apenas uma refeição aos escolares (BRASIL, 2013). Diante do exposto, este trabalho teve como objetivo verificar a quantidade de sal e óleo de adição nas refeições ofertadas aos escolares em uma escola municipal do Estado do Rio de Janeiro.

\section{MÉTODOS}

Este estudo faz parte do projeto de pesquisa "Avaliação das práticas alimentares e do estado nutricional de escolares e identificação de ações e estratégias de promoção, incentivo e/ou apoio à alimentação saudável e atividade física em escolas públicas municipais", aprovado pelo Comitê de Ética em Pesquisa sob o número 0390.0.258.258-11, estando de acordo com a Resolução 196/96 do Conselho Nacional de Saúde. A pesquisa foi realizada após o consentimento da direção da escola.

A coleta dos dados foi realizada durante 12 dias em uma escola municipal do Estado do Rio de Janeiro, sendo determinadas as quantidades de sal e óleo de adição para posterior avaliação das gorduras totais e do sódio presentes nas refeições "almoço" ofertadas, conforme pesquisa realizada por Jackix et al. (2014).

Como as Uanes não dispõem de Fichas Técnicas de Preparo (FTP), foram determinadas as quantidades utilizadas de sal e óleo para cada preparação dos almoços, a partir da diferença entre o peso das quantidades de sal e óleo inicial e final diário disponível para uso na Uane. $O$ sal foi determinado em balança digital da marca Plenna ${ }^{\circledR}$, com precisão de um grama e capacidade para cinco quilos, e o óleo em copo graduado. Após o preparo da refeição do dia pesou-se o rendimento total de cada preparação (arroz, feijão, prato principal e guarnição), descontando-se o peso das panelas, e, posteriormente, foi realizado o somatório do peso total das preparações e estabelecido o percentual de sal/óleo utilizado para todas as preparações presentes nas refeições produzidas naquele dia.

O total de sal/óleo adicionado para preparo das refeições, em quilos, foi dividido pelo peso total das preparações produzidas, em quilos. O percentual total de sal/óleo disponível na refeição produzida foi obtido a partir da Fórmula 1. 
Fórmula 1

\begin{tabular}{|cc|}
\hline $\begin{array}{c}\text { Total de sal/ } \\
\text { óleo } \\
\text { disponível nas } \\
\text { refeições } \\
\text { produzidas }\end{array}$ & $\begin{array}{c}\text { Total de sal/óleo } \\
\text { adicionados para o preparo } \\
\text { das refeições produzidas } \\
(\mathrm{Kg})\end{array}$ \\
\hline & $\begin{array}{c}\text { Total das preparações } \\
\text { produzidas (Kg) } \\
\text { (Arroz + Feijão + Prato } \\
\text { Principal + Guarnição) }\end{array}$ \\
\hline
\end{tabular}

Em seguida foi determinado o peso da refeição ofertada ao escolar por meio de três pesagens da porção de cada preparação, para posterior determinação da gramatura média de cada porção, mediante pesagem de cada preparação alimentar, utilizando-se uma balança digital da marca Plenna ${ }^{\circledR}$, com precisão de uma grama e capacidade para cinco quilos. Desta forma, foi calculada a quantidade de sal/óleo disponível na porção, multiplicando-se a porção da refeição pelo resultado obtido na primeira equação.

Fórmula 2

Sal/óleo disponível na porção = Porção da refeição x Fórmula 1

Para calcular a quantidade de sódio e gorduras totais presentes nas refeições, somou-se o sódio e as gorduras intrínsecos aos alimentos, calculados por meio da Tabela Brasileira de Composição de Alimentos (TACO, 2011), à quantidade dos mesmos presentes no sal e óleo de adição.
As quantidades estimadas de sal e óleo das refeições "almoço" ofertadas foram comparadas com a legislação do PNAE (BRASIL, 2013), que estabelece para uma refeição (no mínimo 20\% das necessidades nutricionais diárias dos alunos matriculados na educação básica), 7,5 g de gorduras totais e $400 \mathrm{mg}$ de sódio.

\section{RESULTADOS}

As preparações alimentares que compuseram as refeições nos 12 dias, a média do porcionamento e a quantidade per capita de sal e óleo de adição na refeição, estão demonstradas na Tabela 1 . Observou-se que em todos os dias analisados os valores de sal de adição na refeição apresentaram-se acima de 1 grama e o óleo adicionado para preparar as refeições teve uma média de $3,6 \mathrm{ml}$.

A Tabela 2 apresenta a quantidade média de sal e óleo de adição por escolar, bem como a quantidade média total encontrada na refeição em relação ao limite máximo de sal e óleo total preconizado pelo PNAE (BRASIL, 2013). O sal de adição está três vezes acima da quantidade permitida, e o conteúdo de gorduras totais (intrínseco e de adição) (12,54 g) encontra-se em níveis bem superiores ao limite máximo recomendado ( $7,5 \mathrm{~g})$, estando este quase duas vezes maior.

Tabela 1 - Porção média da refeição e quantidade per capita de sal $(\mathrm{g})$ e óleo de adição $(\mathrm{ml})$ nas refeições

\begin{tabular}{llccc}
\hline Dia & Preparações do cardápio & $\begin{array}{c}\text { Média da } \\
\text { porção } \\
\text { (g) }\end{array}$ & $\begin{array}{c}\text { Per capita de sal } \\
\text { de adição/escolar } \\
\text { (g) }\end{array}$ & $\begin{array}{c}\text { Óleo de adição/ } \\
\text { escolar (ml) }\end{array}$ \\
\hline $\mathbf{1}$ & Arroz, Feijão, Carne Picada, Chuchu & 310 & 2,47 & 1,80 \\
$\mathbf{2}$ & Arroz, Feijão, Estrogonofe de Carne, Salada de Alface e & 324 & 2,57 & 2,41 \\
& Tomate & 338 & 2,78 & 4,65 \\
$\mathbf{3}$ & Macarrão, Feijão, Coxa Assada, Beterraba & 304 & 1,94 & 3,97 \\
$\mathbf{4}$ & Arroz, Feijão, Carne Seca, Abóbora & 478 & 4,71 & 3,00 \\
$\mathbf{5}$ & Arroz, Feijão, Carne Assada, Cenoura, Tomate e Chuchu & 429 & 4,54 & 3,35 \\
$\mathbf{6}$ & Arroz, Feijão, Carne com Agrião, Berinjela & 218 & 1,54 & 2,07 \\
$\mathbf{7}$ & Arroz, Feijão, Isca de Frango & 392 & 2,64 & 6,40 \\
$\mathbf{8}$ & Macarrão, Feijão, Ovo cozido & 449 & 3,07 & 4,14 \\
$\mathbf{9}$ & Arroz, Feijão, Isca de Frango, Abobrinha & 379 & 4,08 & 3,09 \\
$\mathbf{1 0}$ & Arroz, Feijão, Sobrecoxa de Frango, Quiabo, Salada de Alface & 394 & 3,90 \\
$\mathbf{1 1}$ & Arroz, Feijão, Músculo, Salada de Beterraba & 301 & 5,17 \\
$\mathbf{1 2}$ & Arroz, Feijão, Atum, Purê de Batata & 401 & 4,15 & 3,72 \\
& Média & 144,82 & 3,06 & 1,06 \\
\hline
\end{tabular}


Tabela 2 - Quantidade média de sal e óleo adicionados às preparações em relação ao conteúdo total (somatória entre o sal/óleo de adição e o sódio/lipídios presente naturalmente nos alimentos) e ao limite de sal e óleo total preconizado pela Resolução no 26 do PNAE de 2013 (BRASIL, 2013)

\begin{tabular}{ccccc}
\hline Dia & $\begin{array}{c}\text { Per capita de sal de } \\
\text { adição/escolar } \mathbf{( g )}\end{array}$ & $\begin{array}{c}\text { Sódio Total na } \\
\text { refeição }(\mathbf{m g})\end{array}$ & $\begin{array}{c}\text { Óleo de adição/ } \\
\text { escolar }(\mathbf{m l})\end{array}$ & $\begin{array}{c}\text { Lipídios na refeição } \\
\mathbf{( g )}\end{array}$ \\
\hline $\mathbf{1}$ & 2,47 & 1036,95 & 1,80 & 12,21 \\
$\mathbf{2}$ & 2,57 & 1072,50 & 2,41 & 11,70 \\
$\mathbf{3}$ & 2,78 & 1177,79 & 4,65 & 12,17 \\
$\mathbf{4}$ & 1,94 & 2532,70 & 3,97 & 16,66 \\
$\mathbf{5}$ & 4,71 & 1924,17 & 3,00 & 11,14 \\
$\mathbf{6}$ & 4,54 & 1869,76 & 3,35 & 14,82 \\
$\mathbf{7}$ & 1,54 & 637,04 & 2,07 & 4,37 \\
$\mathbf{8}$ & 2,64 & 1150,17 & 6,40 & 14,97 \\
$\mathbf{9}$ & 3,07 & 1261,94 & 4,14 & 8,00 \\
$\mathbf{1 0}$ & 4,08 & 1712,89 & 3,09 & 12,92 \\
$\mathbf{1 1}$ & 2,17 & 922,51 & 3,90 & 8,75 \\
$\mathbf{1 2}$ & 4,15 & 2612,92 & 5,85 & 22,80 \\
Recomendação (PNAE) & 1,00 & 400,00 & 7,50 & 7,50 \\
Média & 3,06 & 1492,61 & 3,72 & 12,54 \\
Desvio Padrão & 1,06 & 634,22 & 1,42 & 4,65 \\
\hline
\end{tabular}

Fonte: Elaborada pelas autoras.

\section{DISCUSSÃO}

Diversos estudos apontam para a influência de padrões alimentares específicos no surgimento de obesidade/sobrepeso e de outras doenças crônicas não transmissíveis (DCNT) (RONCA et al., 2019; ARAUJO et al., 2017; SOUZA et al., 2016). Desta forma, deve-se incentivar e promover o consumo de alimentos in natura ou minimamente processados e de refeições preparadas com esses alimentos, e desencorajar o consumo de alimentos processados e ultraprocessados. $\mathrm{O}$ uso dos ingredientes culinários, como sal, óleos e açúcares, no entanto, deve ser feito de maneira criteriosa, especificamente quando o público-alvo são as crianças (BRASIL, 2014a; MONTEIRO et al., 2016).

A legislação do PNAE considera que os alimentos processados e ultraprocessados, geralmente muito ricos nestes ingredientes, podem ser utilizados desde que de maneira restrita na alimentação escolar, isto é, atendendo ao limite máximo de $30 \%$ para a aquisição desses alimentos (BRASIL, 2013). Nesse sentido, importa destacar que os teores mais elevados de sódio foram encontrados neste estudo nos dias $4(2532,7$ $\mathrm{mg}$ ) e $12(2612,9 \mathrm{mg})$, em que alimentos processados, como carne seca e atum enlatado, foram oferecidos no cardápio (Tabela 2). Considerando que a legislação do PNAE determina limites de açúcar, sódio e gorduras totais, saturadas e trans, nutrientes presentes em grande proporção em alimentos processados, propõe-se que a seleção de alimentos para o PNAE considere de modo privilegiado o seu grau de processamento, conforme o recomendado pelo Guia Alimentar para a População Brasileira (BRASIL, 2014a; MONTEIRO et al., 2016).

O peso da porção das refeições avaliadas variou de $218 \mathrm{~g}$ até $801 \mathrm{~g}$, posto que esta variação pode ser atribuída à falta de padronização das porções alimentares oferecidas aos escolares. O tamanho da porção oferecida pode influenciar diretamente na quantidade de lipídios ofertados, dificultando a adequação de energia, macro e micronutrientes, podendo trazer riscos à saúde dos escolares. Assim, os estudos apontam para a necessidade de padronizar as preparações alimentares de acordo com a faixa etária e capacitar as merendeiras para realizar a distribuição adequada das porções servidas às crianças (BEZERRA; CORADI, 2016; MOREIRA et al., 2017; MENEZES; SANTANA; NASCIMENTO, 2018).

Em todos os dias analisados, o sal de adição excedeu a recomendação de 1 grama por refeição, posto que a média de sal adicionado às refeições foi três vezes maior que a recomendação do PNAE. A ausência de FTP é uma realidade comum nas Uanes, sendo considerado um item importante para o controle de açúcares, sal e óleo de adição no preparo das refeições para os escolares (SOUZA; MARSI, 2015). Cabe desta- 
car que a quantidade de sódio em dois dias ultrapassa o valor máximo diário recomendado pela OMS (WHO, 2015), que é de $2.000 \mathrm{mg}$ de sódio.

No presente estudo, o sal de adição representou $82 \%$ do total de sódio presente na refeição, uma vez que a média de sódio de adição nas refeições foi de $1.222 \mathrm{mg}$ e a média de sódio total nas refeições de $1.492,6 \mathrm{mg}$. Este resultado corrobora o estudo de Legetic e Campbell (2011), que verificaram que pelo menos $70 \%$ do sal total, presente nas refeições, provém de sua adição às preparações alimentares. Desta forma, evidencia-se a necessidade da supervisão do processo produtivo de refeições pelo nutricionista, responsável técnico pelas Uane.

O Guia de Boas Práticas Nutricionais, visando à orientação dos serviços de alimentação quanto ao preparo de alimentos com menores teores de açúcar, gorduras e sódio, foi desenvolvido pela Agência Nacional de Vigilância Sanitária (BRASIL, 2014b). Essa prática do controle da adição desses ingredientes, no entanto, ainda apresenta desafios, destacando a conscientização do manipulador dos alimentos e do consumidor e a elaboração de FTPs mais práticas e adequadas à realidade desses manipuladores de alimentos.

A OMS lidera uma mobilização mundial para a redução da ingestão de sódio, a fim de minimizar efeitos do seu consumo excessivo (WHO, 2015). Nas Américas, a Organização Pan-americana de Saúde propôs uma força tarefa para redução desse consumo, e muitos países, incluindo o Brasil, traçaram estratégias para alcançar essa meta proposta até o ano de 2020, com a redução do consumo de sal para menos de $5 \mathrm{~g} /$ dia, o que corresponde a $2.000 \mathrm{mg}$ de sódio (NILSON; JAIME; RESENDE, 2012). Para a efetivação da meta nacional de redução do consumo de sal pela população brasileira, o Ministério da Saúde vem realizando medidas educativas para o aumento do consumo de alimento in natura e articulando com o setor produtivo de alimentos a redução voluntária dos teores de sódio em alimentos processados e ultraprocessados (BRASIL, 2018).

Como o cloreto de sódio é um estimulador do apetite e um modulador do sabor, crianças adquirem o gosto por sal conforme a quantidade que ingerem diariamente (JACKIX et al., 2014; LONGO-SILVA, 2014). Desta forma, o responsável técnico pela alimentação escolar deve conscientizar e sensibilizar as merendeiras para a padronização das técnicas de cocção, especialmente na redução do sal de adição, considerando que, com o intuito de aumentar a aceitação por parte das crianças, as merendeiras podem adicionar gran- des quantidades de sal às preparações (SANTOS; SREBERNICH, 2015; BEZERRA; CORADI, 2016; ROCHA et al., 2018).

Segundo estudos, os consumidores dificilmente notam uma redução de sódio de até $30 \%$ se esta for feita de forma gradual durante o preparo dos alimentos (BRASIL, 2014b). Ao tentar reduzir o consumo, todavia, pessoas habituadas a alimentos salgados tendem a considerar o sabor dos alimentos não tão satisfatórios. As células do paladar, contudo, podem levar até três meses para se ajustar ao sabor menos intenso do sal, sendo importante esse esclarecimento às merendeiras para persistirem no preparo de refeições com menor teor de sal (RANNOU et al., 2018). A priorização de técnicas culinárias mais adequadas, privilegiando o uso de temperos naturais, como cebola, alho e ervas aromáticas, é uma grande aliada nesse processo (LONGO-SILVA et al., 2014), uma vez que favorece a diminuição da quantidade de sal utilizada para o preparo das refeições, sem que ocorra prejuízo no sabor.

A quantidade de óleo de adição representou em média $29,7 \%(3,72 \mathrm{~g})$ dos lipídios ofertados na refeição $(12,54 \mathrm{~g})$. Ou seja, a maior parte dos lipídios era proveniente da composição dos alimentos e não do óleo adicionado durante o preparo. Não existem estudos que orientem quanto à quantidade de adição de óleo durante o preparo dos alimentos, sabendo-se apenas a quantidade de lipídios recomendadas por refeição. Como observado na Tabela 2, a média de lipídios ultrapassou o limite recomendado por refeição, que seria de $7,5 \mathrm{~g}$, em quase o dobro $(12,54 \mathrm{~g})$.

Em apenas um dia de estudo, o total de lipídios ficou abaixo de 7,5g, e no restante dos dias variou de $8,0 \mathrm{~g}$ até $22,8 \mathrm{~g}$ na refeição. Alguns autores evidenciaram resultados semelhantes em relação à quantidade de lipídios nas refeições escolares (BEZERRA; CORADI, 2016; BEZ, 2017; SOUZA et al., 2018; ROCHA et al., 2018). Preparações com alto teor de lipídios contribuem para o aumento do valor energético da refeição oferecida, o que confirma a necessidade de analisar constantemente o conteúdo de lipídios presentes nos alimentos durante o planejamento do cardápio, pois muitas crianças realizam suas principais refeições nas escolas, e o elevado consumo diário pode desencadear o desenvolvimento de obesidade e dislipidemias.

A disparidade entre os valores de sódio e óleo nas preparações pode ser justificada pela ausência de FTP, aliado a um modo especial de tempero não padronizado, baseado, muitas vezes, no paladar das merendeiras que são responsáveis pela elaboração das refeições (SANTOS; SREBERNICH, 2015; RETONDARIO 
et al., 2015). A padronização com ênfase na redução dos teores de sal e óleo de adição para cocção das preparações, seria bastante positiva para uma meIhora na qualidade das refeições oferecidas aos escolares. Cabe destacar que este estudo analisou apenas a quantidade de sal e óleo disponíveis nas refeições servidas, não sendo avaliada a ingestão alimentar das crianças, uma vez que os desperdícios não foram contabilizados. Este fato deve ser levado em consideração para a interpretação dos resultados.

\section{CONCLUSÕES}

$O$ estudo demonstrou que a quantidade média de sódio (adição e intrínseco) por refeição ultrapassou o que é preconizado pelo PNAE. Essa oferta excessiva pode ser reduzida por meio de ações corretivas, como a capacitação das merendeiras para redução do uso do sal de adição e a elaboração de FTP para padronização das preparações. Faz-se necessária a realização de ações educativas incentivando a redução do consumo de sódio dentro e fora do ambiente escolar para a promoção de hábitos alimentares saudáveis. Ressalta-se que a conscientização e a sensibilização das merendeiras são fundamentais para a adequação das refeições quanto ao teor de sódio e outros ingredientes adicionados.

Em razão da alta quantidade de lipídios oferecidos aos escolares nas refeições, o estudo também aponta para a necessidade de um acompanhamento mais efetivo por parte do nutricionista dentro da escola e no planejamento do cardápio para manter o limite recomendável deste nutriente.

\section{REFERÊNCIAS}

ARAUJO, M. C.; CUNHA, D. B.; BEZERRA, I. N.; CASTRO, M.B.T.; SICHIERI, R. Quality of food choices of Brazilian adolescents according to individual earnings. Public Health $\mathrm{Nu}$ trition, Cambridge, v. 20, n. 17, p. 3.145-3.150, 2017.

BEZ, A. Aceitação da alimentação e análise do cardápio escolar de uma Escola Municipal de Francisco Beltrão - PR. Revista da Associação Brasileira de Nutrição, ano 8, n. 2, p. 12-19, 2017.

BEZERRA, A.; CORADI, F. Análise da alimentação oferecida para crianças em uma creche municipal do Rio Grande do Sul. Revista Contexto \& Saúde, ljuí: Editora Unijuí, v. 16, n. 31, p. 56-69, 2016.

BOUHLAL, S.; ISSANCHOU, S.; NICKLAUS, D. The impact of salt, fat and sugar levels on toddler food intake. British Journal Nutrition, v. 105, n. 4, p. 645-53, 2011.

BRASIL. Ministério da Educação e Cultura. Fundo Nacional de Desenvolvimento da Educação. Resolução/CD/FNDE ${ }^{\circ}$ 26, de 17 de junho de 2013. 2013. Dispõe sobre o atendi- mento da alimentação escolar aos alunos da educação básica no âmbito do Programa Nacional de Alimentação Escolar-PNAE. Brasília: MEC; FNDE, 2013.

BRASIL. Ministério da Saúde. Secretaria de Atenção à Saúde. Departamento de Atenção Básica. Guia alimentar para a população brasileira. 2. ed. Brasília: Ministério da Saúde, 2014a. $156 \mathrm{p}$.

BRASIL. Ministério da Saúde. Agência Nacional de Vigilância Sanitária. Guia de Boas Práticas Nutricionais. Brasília: Ministério da Saúde, 2014b.

BRASIL. Ministério da Saúde. Secretaria de Atenção à Saúde. Departamento de Atenção Básica. Monitoramento do Plano Nacional de Redução do Sódio em Alimentos Processados. Brasília: Ministério da Saúde, 2018.

IBGE. Instituto Brasileiro de Geografia e Estatística. Pesquisa de orçamentos familiares 2008-2009: análise do consumo alimentar no Brasil. Rio de Janeiro: IBGE, 2011.

JACKIX, E. A.; BORREGO, F.; STENICO, A. R.; SAVITSKY, J. P.; CORDOBA, G. M. C. Avaliação da quantidade de óleo e sal adicionados à alimentação escolar em creches da cidade de Nova Odessa-SP. Ciência \& Inovação, v. 1, n. 1, p. 7, 2014.

LEGETIC, B.; CAMPBELL, N. Reducing salt intake in the Americas: Pan American Health Organization actions. Journal Health Communication, v. 16, n. 2, p. 37-48, 2011.

LONGO-SILVA, G.; TOLONI, M. H. A.; MENEZES, R. C. E.; TEMTEO, T. L.; OLIVEIRA, M. A. A.; ASAKURA, L. Ingestão de proteína, cálcio e sódio em creches públicas. Revista Paulista de Pediatria, São Paulo, v. 32, n. 2, p. 193-199, 2014.

LOUZADA, M. L. C.; MARTINS, A. P. B.; CANELLA, D. S.; BARALDI, L. G.; LEVY, R. B.; CLARO, R. M.; MOUBARAC, J. C.; CANNON, G.; MONTEIRO, C. A. Alimentos ultraprocessados e perfil nutricional da dieta no Brasil. Revista de Saúde Pública, v. 49, n. 38, 2015.

MILL, J. G. et al. Estimativa do consumo de sal pela população brasileira: resultado da Pesquisa Nacional de Saúde 2013. Revista Brasileira de Epidemiologia, v. 22, supl. 2, E190009, 2019.

MENEZES, R. O. S; SANTANA, E. M.; NASCIMENTO, M. O. L. elaboração de fichas técnicas das preparações oferecidas em serviço de alimentação e nutrição de hospital público de Salvador, BA. Higiene Alimentar, v. 32, n. 284-285, set./ out. 2018.

MONTEIRO, C. A.; CANNON, G.; LEVY, R. B. MOUBARAC, J. C.; JAIME, P.; MARTINS, A. M.; CANELLA, D.; LOUZADA, M.; PARRA, D. Nova. The star shines bright. Food classification. Public health. World Nutrition, v. 7, n. 1-3, p. 28-38, 2016.

MOREIRA, R.; COUTINHO, M. B. C.; MENDONÇA, D.; BASTOS, D. S.; HENRIQUES, P.; CAMACHO, P. School nutrition program: Assessment of planning and nutritional recommendations of menus. Revista Chilena Nutrición, v. 44, n. 2, p. 170-176, 2017.

NILSON, E. A. F.; JAIME, P. C.; RESENDE, D. O. Iniciativas desenvolvidas no Brasil para a redução do teor de sódio em alimentos processado. Revista Panamericana de Salud Publica, v. 34, n. 4, p. 287-92, 2012. 
RANNOU, C.; TEXIERA, F.; MARZIN, C.; NICKLAUS, S.; CARIOU, V.; COURCOUX, P.; PROST, C. Effect of Salt Reduction on Children's Acceptance of Bread. Journal of food science, $v$. 83, n. 8, p. 2.204-2.211, 2018.

RETONDARIO, A.; SILVA, D. L. F; ALVES, M. A. O.; ALMEIDA, C. C. B.; SCHMIDT, S. T.; FERREIRA, S. M. R. Oferta de sódio na alimentação em creches municipais: um risco à saúde. Mundo Saúde, v. 39, n. 1, p. 11-21, 2015.

ROCHA, N. P.; FILGUEIRAS, M. S.; ALBUQUERQUE, F. M.; MILAGRES, L. C.; CASTRO, A. P. P.; SILVA, M. A.; COSTA, G. D.; PRIORE, S. E.; NOVAES, J. F. Análise do programa nacional de alimentação escolar no município de Viçosa, MG, Brasil Revista de Saúde Pública, v. 52, n. 16, 2018.

RONCA, D. B.; BLUME, C. A.; CUREAU, F. V.; CAMSEY, S. A.; LEOTTI, V. B.; DREHMER, M. Diet quality index for Brazilian adolescents: the ERICA study. European Journal of Nutrition, v. 1, n. 18, 2019.

SANTOS, A. R.; SREBERNICH, S. M. Determinação do teor de sódio em preparações de arroz, feijão e carne servidas nas Escolas Municipais de Ensino Infantil (EMEIS) da Região Sul do município de Campinas. ENCONTRO DE INICIAÇÃO CIENTÍFICA, 20., ENCONTRO DE INICIAÇÃO EM DESENVOLVIMENTO TECNOLÓGICO E INOVAÇÃO, 5. 2015, Campinas. São Paulo: PUC Campinas, 22-23 set. 2015.

SILVA, G. A. P.; COSTA, K. A. O.; GIUGLIANI, E. R. J. Alimentação infantil: além dos aspectos nutricionais. Jornal de Pediatria, v. 92, n. 3, supl. 1, p. 2-7, 2016.

SOUZA, A. M.; BARUFALDI, L. A.; ABREU, G. A.; GIANNINI, D. T.; OLIVEIRA, C. L.; SANTOS, M. M. Erica: Intake of macro and micronutrients of Brazilian adolescents. Revista de Saúde Pública, v. 50, n. supl 1, p. 1s-15s, 2016.

SOUZA, L. V.; MARSI, C. O. T. Importance of technical in UANs: production and costs preparations/meals. Journal of the Health Sciences Institute, v. 33, n. 3, p. 248-253, 2015.

SOUZA, C. A. N.; LONGO-SILVA, G.; MENEZES, R. C. E.; ARAUJO, A. C.; TOLONI, M. H. A.; OLIVEIRA, M. A. A. Adequação nutricional e desperdício de alimentos em Centros de Educação Infantil. Ciência \& Saúde Coletiva, v. 23, n. 12, p. 4.177-4.188, 2018.

TACO. Tabela Brasileira de Composição de Alimentos. 4. ed. Campinas: Nepa; Unicamp, 2011.

WHO. World Health Organization. 2015. Guideline: Sugars intake for adults and children, 2015. Disponível em: http:// who.int/nutrition/publications/guidelines/sugars_intake/ en/. Acesso em: 1ㅇfev. 2020. 